\title{
Correction to: Adjuvant Chemotherapy for Gastric Cancer Patients with Mismatch Repair Deficiency or Microsatellite Instability: Systematic Review and Meta-Analysis
}

\author{
Run Cong Nie ${ }^{1}$, Guo Ming Chen ${ }^{1}$, Shu Qiang Yuan, Jin Won Kim², Jie Zhou ${ }^{3}$, Man Nie ${ }^{4}$, \\ Chen Yang Feng ${ }^{5}$, Ying Bo Chen ${ }^{1}$, Shi Chen ${ }^{6}$, Zhi Wei Zhou ${ }^{1}$, Yun Wang ${ }^{7}$, and Yuan Fang Li $^{1}$ \\ ${ }^{1}$ Department of Gastric Surgery, State Key Laboratory of Oncology in South China, Collaborative Innovation Center for \\ Cancer Medicine, Sun Yat-sen University Cancer Center, Guangzhou, People's Republic of China; ${ }^{2}$ Division of \\ Hematology and Medical Oncology, Department of Internal Medicine, Seoul National University Bundang Hospital, Seoul \\ National University College of Medicine, Seongnam, Korea; ${ }^{3}$ State Key Laboratory of Oncology in South China, \\ Collaborative Innovation Center for Cancer Medicine, Sun Yat-sen University Cancer Center, Guangzhou, People's \\ Republic of China; ${ }^{4}$ Department of Medical Oncology, State Key Laboratory of Oncology in South China, Collaborative \\ Innovation Center for Cancer Medicine, Sun Yat-sen University Cancer Center, Guangzhou, People's Republic of China; \\ ${ }^{5}$ Department of Information, State Key Laboratory of Oncology in South China, Collaborative Innovation Center for \\ Cancer Medicine, Sun Yat-sen University Cancer Center, Guangzhou, People's Republic of China; ${ }^{6}$ Department of \\ Gastrointestinal Surgery, the Sixth Affiliated Hospital, Sun Yat-sen University, Guangzhou, People's Republic of China; \\ ${ }^{7}$ Department of Hematologic Oncology, State Key Laboratory of Oncology in South China, Collaborative Innovation \\ Center for Cancer Medicine, Sun Yat-sen University Cancer Center, Guangzhou, People's Republic of China
}

\section{CORRECTION TO: ANN SURG ONCOL HTTPS://DOI.ORG/10.1245/S10434-021-11050-6}

The following article notes have been added to the original online version of the article:

R.C. Nie, G.M. Chen, S.Q. Yuan, J.W. Kim, J. Zhou and M. Nie contributed equally to this study.
Z.W. Zhou, Y. Wang, and Y.F. Li are co-senior authors.

Publisher's Note Springer Nature remains neutral with regard to jurisdictional claims in published maps and institutional affiliations.

R.C. Nie, G.M. Chen, S.Q. Yuan, J.W. Kim, J. Zhou and M. Nie contributed equally to this study.

Z.W. Zhou, Y. Wang, and Y.F. Li are co-senior authors.

The original article can be found online at https://doi.org/10.1245/ s10434-021-11050-6.

(C) Society of Surgical Oncology 2021

Published online: 6 December 2021

Y. F. Li

e-mail: zhouzhw@sysucc.org.cn 